\title{
Treatment approaches of electrical injuries
}

\author{
Anatolie Taran \\ Department of Orthopedics and Traumatology \\ Nicolae Testemitanu State University of Medicine and Pharmacy, Chisinau, the Republic of Moldova
}

Author's ORCID iD, academic degrees and contributions are available at the end of the article

Corresponding author - Anatolie Taran, e-mail: anatol_taran@yahoo.com

Manuscript received October 15, 2021; revised manuscript November 09, 2021; published online November 26, 2021

\begin{abstract}
Background: The purpose of this study was to explore the differences in prehospital care, admission characteristics, burn intensive care, surgery and outcomes in patients requiring admission to a burn intensive care unit.

Material and methods: The study was conducted on a group of 31 patients, who were hospitalized within the Clinical Hospital of Orthopedics and Traumatology in the period 2015-2019. The data analysis was carried out on the applied method of surgical treatment, the associated postoperative complications, antibiotic therapy applied, etc.

Results: 27 out of the total number of patients underwent necrectomy and extensive wound debridement surgery in the first hours upon admission. 14 patients required additional decompression incisions in the underlying skin and fascia. 4 patients out of the total number presented visible signs of carbonization on the affected areas upon admission, therefore they were subjected to an emergency amputation. Out of 27 patients classified as "delayed emergency" cases, 13 patients were subjected to amputation of the corresponding segments.

Conclusions: Electrical injuries are a severe cause of disability, as well as a challenging issue for reconstructive surgery, which is concerned with restoring the damaged structures with prosthetic amputation abutments, therefore improving the aesthetic and psychological appearance of the patients.

Key words: electrical injury, electric shock, reconstructive surgery, high and low voltage burn injuries.
\end{abstract}

Cite this article

Taran A. Treatment approaches of electrical injuries. Mold Med J. 2021;64(5):38-41. https://doi.org/10.52418/moldovan-med-j.64-5.21.07.

\section{Introduction}

Electrical injuries are classified as extremely serious injuries, commonly resulting in a poor life and poor functional prognosis, which can only be improved by complex general and local treatment, tailored to the individual characteristics of each case while considering the extreme urgency [1]. The prognosis of electrical burns depends on various factors:

- Burn surface area;

- Degree of the burn;

- Age;

- Possible complications of the burn;

- Patient's health before the accident;

- Occurrence of concomitant injuries (particularly, lung injuries);

- Early treatment and quality of treatment [2].

The only factor that can be corrected from the aforementioned ones is the "early and quality treatment" for the patient with an electric burn being admitted to a medical facility, thus the present issue requires an in-depth research approach.

The electric burn treatment should start immediately after it occurred and continue during the recovery period [2]. Any electric burn is an immediate surgical emergency, hence the surgical treatment will be carried out together with other drug therapies, which will significantly increase the chances of a quality recovery [2].

Successful treatment is based on a detailed clinical examination, which is actually a real strategy in this case, requiring knowledge on the multiple peculiarities of this type of lesion [3]. During the clinical examination, special attention is focused on the following areas:

- Electric entry mark and electric exit mark;

- Injuries caused by the electric path;

- Thermal burns caused by electric flames or ignition of clothing [3].

Following a complete initial assessment of the affected lesions, emergency treatment will be initiated. Local treatment involves a surgical intervention which is a critical component in the acute phase of electrical shock [4]. Emergency surgery is aimed at removing dead tissues and cleaning the associated burn injuries, on the one hand, as well as clearing the blood flow by preventing or combating the development of acute peripheral ischemia syndrome, on the other hand [1].

Necrectomy and extensive wound debridement are the primary goal of local treatment. Basically, the extended pressure sore excision and deep cleansing of devitalized tis- 
sues might eliminate the main source of toxic substances (myoglobin, denatured proteins, cellular metabolites), as well as all the sepsis-causing bacteria [1]. Although a conservative treatment is commonly applied, in cases when sepsis can be life-threatening, the amputation will be performed as quickly as possible. Direct trauma in electrical injuries causes gradual ischemia, forming thrombi in the small artery or arteriole, constriction of blood vessels slowing blood flow and causing tissue necrosis, being a decision factor for amputation. To protect viable tissue in the initial stage of treatment is fasciotomy decompression of the involved compartment. The next step is to approach the methods of reconstruction from simple to compound by using skin grafts, already regional or free flaps need to be used in severe lesions (local flaps, removed flaps and free flaps). The local treatment should be combined with general resuscitation treatment aiming to implementing therapeutic shock recovery and rebalancing the hemo-hydro-electrolytic levels, on the one hand, as well as preventing or managing complications, on the other hand $[5,6]$.

In this context, the present research paper aims to highlight the peculiarities of the clinical evolution of the patients after electrical injuries depending on the surgical strategies used.

\section{Material and methods}

The study group involved 31 patients admitted to hospital in the period 2015-2019 at the Clinical Hospital of Traumatology and Orthopaedics.

The main study inclusion criterion was the electrical injury.

The characteristics determined for each patient comprised:

- Age;

- Gender distribution;

- The surgical approach applied;

- Antibiotic therapy;

- Length of hospital stay;

- Associated complications.

\section{Results}

By gender distribution, the groups were divided as follows: 21 cases $(67.74 \%)$ - men and 10 cases $(32.25 \%)$ - women (fig. 1). Of the total number of 31 cases: 4 cases (12.9\%) - B third-burn degree, 7 cases (22.58\%) - AB thirdburn degree and 20 cases (64.51\%) - fourth-burn degree (fig. 2).

The distribution of hospitalized patients by age groups was as follows: $21-45$ years - $11(35.48 \%)$ patients, $46-60$ years - $17(54.83 \%)$ patients, over 60 years - $3(9.67 \%)$ patients (fig. 3 ).

Upon the first hours after hospitalization, 27 patients (87.09\%) out of 31 underwent necrectomy and extensive wound debridement. In the other 4 cases (12.9\%) radical intervention was performed by emergency amputation (fig.
4). Out of 4 patients who were amputated upon the first hours of hospitalization, the lower limb was amputated in 3 cases and the upper limb was amputated in only one case.

Of the 27 patients who underwent necrectomy and surgical wound debridement upon the first hours of hospitalization, 8 patients lost their sensory function and pulse in the distal part of the limb during the following hours, whereas the other 6 patients had an increased limb circumference due to massive edema. Thus, 14 out of 27 cases required additional decompression incisions in the underlying skin and fascia.

Finally, of the 27 patients categorized as delayed emergency cases, a decision on segment amputation was made in 13 cases.

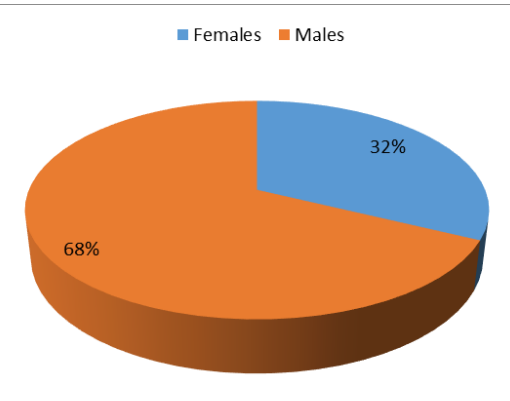

Fig. 1. Gender distribution of patients

— B third-burn degree $\quad$ AB third-burn degree $\quad$ - fourth-burn degree

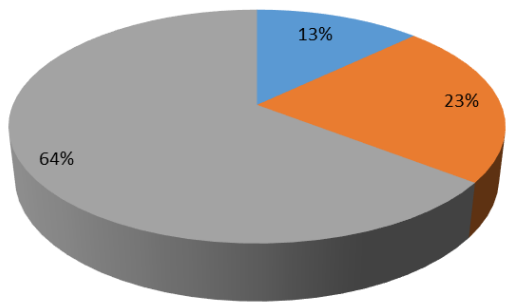

Fig. 2. Distribution of patients by burn degree

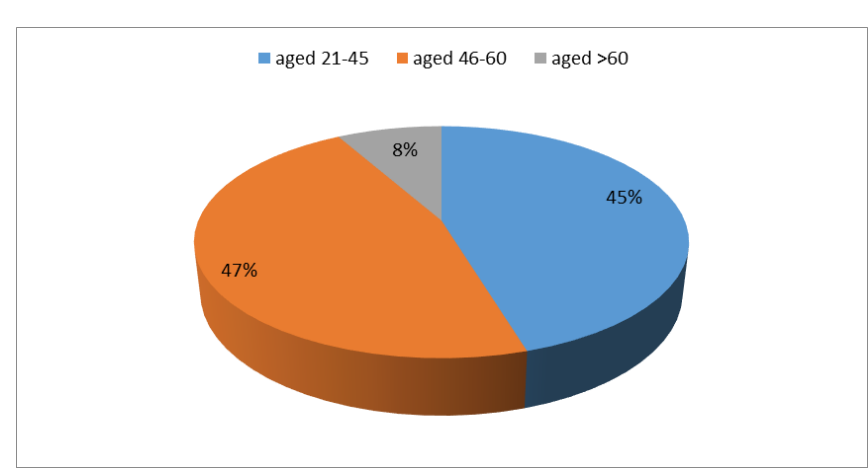

Fig. 3. Distribution of patients by age groups 


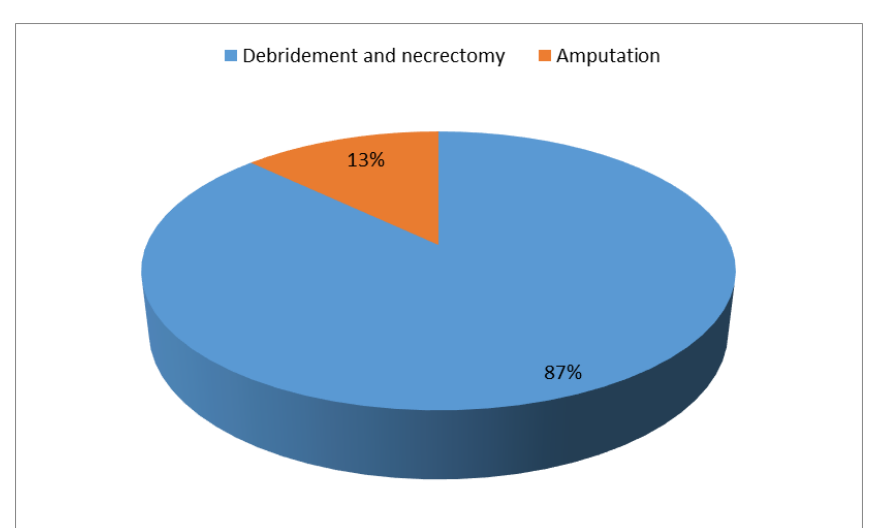

Fig. 4. Distribution of patients by type of intervention performed upon the first hours of admission

Out of 13 patients who managed to save the limb affected by the electric shock, a deficiency of optimal wound coverage was reported in 9 cases, therefore epithelization was stimulated via skin and flap autografts.

The average length of hospital stay was 33.7 days.

As regarding the antibiotic therapy, two or more associated antibiotics were administered in all 31 cases. The primary purpose was the administration of cephalosporins combined with fluoroquinolones (levofloxacin) or betalactam penicillin group (amoxicillin). To cover the group of anaerobic bacteria, Metronidazole was administered in all cases.

Complications associated with electrical injuries were grouped into the following categories: acute renal failure - 5 cases; atrial fibrillation -3 cases; atrioventricular block - 2 cases; pneumonia - 2 cases.

\section{Discussion}

Treatment of electrical injuries is difficult, complex and should be tailored according to the particularities of the patient. The effectiveness of early decompression incisions, necrectomies and surgical debridement has been proven, thus ensuring the removal of necrotic detritus, relieving skin tension, improving blood flow, including peripheral blood circulation and eliminating edema. However, the procedures were not adequate for all cases, thus requiring additional decompression incisions. For this reason, it was concluded that a longer time of vascular compromise might lead to greater incidence of deep subfascial edema, similar to crush syndrome. Along with decompression incisions, deep exploration incisions are also important for assessing the condition of neurovascular elements, bones and muscles.

Regardless of the voltage that caused the electric burn (low or high), patients should be treated in the same way as for high voltage injuries as they may manifest as loss of consciousness, compartment syndrome, myoglobinuria and hemoglobinuria [7].

In 4 cases, when emergency amputation was performed, the patients showed signs of limb carbonization even upon admission to medical institution, therefore, appropriate interventions were performed to avoid life-threatening risks. The "delayed emergency" cases resulted in amputations due to the patient's unfavorable clinical development, as well as being associated with septic complications and progression to wet gangrene of the corresponding limb.

Electrical injuries are the result of preventable accidents. The transformation of electricity into thermal energy through the direct effect in association with blunt mechanical trauma can lead to tissue destruction and organ dysfunction, thus requiring trauma management and thorough clinical management, which will establish the stages of surgical treatment in several stages (fasciotomy, skin graft, long-term supportive care) [8].

The optimal wound coverage is another key point in the treatment of electrical injuries, since it should be performed as early as possible to prevent superinfection of the wound accompanied by secondary necrosis.

\section{Conclusions}

1. Electrical injuries are serious emergencies that can lead to patient's death unless proper medical or surgical management is provided.

2. The patient suffering from an electric shock should be treated comprehensively and by a multidisciplinary team, in order to prevent life-threatening conditions due to both direct consequences of electrical injury and commonly associated general complications.

3. The surgical intervention is the treatment of choice, however if patients present all the indications for segment amputation, it should be inevitably performed so as not to endanger the patient's life.

4. Treatment of a patient with electrical injury continues despite the correction of the underlying problem. It continues during the rehabilitation period and involves numerous reconstructive operations aimed at restoring the damaged structures and prosthesis of amputation abutments, as well as improving the aesthetic appearance.

\section{References}

1. Isac F, Brezeanu C. Leziunile prin curent electric [Injuries by electric current]. In: Angelescu N, editor. Tratat de patologie chirurgicala [Treatise on surgical pathology]. Bucharest: Editura Medicala; 2001. p. 704-710. Romanian.

2. Brătucu E, editor. Manual de chirurgie pentru studenți [Surgery manual for students]. Bucharest: Editura Universitară “Carol Davila”, 2009. Romanian.

3. Mugea TT. Agresiuni termoenergetice [Thermoenergetic lesions]. Oradea: Editura Universității din Oradea; 2002. p. 225-289; p. 336-352. Romanian.

4. Isac F, Isac A, Bratu T, Tache M, Draghici I. Arsurile [Burns]. Timişoara: Dinamis Print; 1994. p. 142-145. Romanian.

5. Dechent D, Emonds T, Stunder D, Schmiedchen K, Kraus T, Driessen S. Direct current electrical injuries: a systematic review of case reports and case series. Burns. 2020;46(2):267-278. doi: 10.1016/j.burns.2018.11.020.

6. Kym D, Seo DK, Hur GY, Lee JW. Epidemiology of electrical injury: differences between low- and high-voltage electrical injuries during a 
7-year study period in South Korea. Scand J Surg. 2015;104(2):108-114 doi: 10.1177/1457496914534209.

7. Yastı AÇ, Şenel E, Saydam M, Özok G, Çoruh A, Yorgancı K. Guideline and treatment algorithm for burn injuries. Ulus Travma Acil Cerrahi Derg. 2015;21(2):79-89. doi: 10.5505/tjtes.2015.88261.
8. Pinto DS, Clardy PF, Moreira ME. Environmental and weapon-related electrical injuries. UpToDate [Internet]. 2016. [cited 2021 May 30]. Available from: https://www.uptodate.com/contents/environmentaland-weapon-related-electrical-injuries

\section{Author's ORCID iD and academic degrees}

Anatolie Taran, MD, PhD, Professor - https://orcid.org/0000-0002-8778-0200

\section{Author's contributions}

AT conceptualized the project, designed the research, interpreted the data and wrote the manuscript.

\section{Funding}

This study was supported by Nicolae Testemitanu State University of Medicine and Pharmacy. The trial was the author's initiative. The author is independent and takes responsibility for the integrity of the data and accuracy of the data analysis.

\section{Ethics approval and consent to participate}

The research project was approved by the Research Ethics Committee of Nicolae Testemitanu State University of Medicine and Pharmacy (Protocol No 2 of 23.10.2017).

\section{Conflict of Interests}

No competing interests were disclosed. 